ACCEPTED MANUSCRIPT

\title{
Exploring Event Horizons and Hawking Radiation through Deformed Graphene Membranes
}

To cite this article before publication: Tommaso Morresi et al 2020 2D Mater. in press https://doi.org/10.1088/2053-1583/aba448

\section{Manuscript version: Accepted Manuscript}

Accepted Manuscript is "the version of the article accepted for publication including all changes made as a result of the peer review process, and which may also include the addition to the article by IOP Publishing of a header, an article ID, a cover sheet and/or an 'Accepted

Manuscript' watermark, but excluding any other editing, typesetting or other changes made by IOP Publishing and/or its licensors"

This Accepted Manuscript is @ 2020 IOP Publishing Ltd.

During the embargo period (the 12 month period from the publication of the Version of Record of this article), the Accepted Manuscript is fully protected by copyright and cannot be reused or reposted elsewhere.

As the Version of Record of this article is going to be / has been published on a subscription basis, this Accepted Manuscript is available for reuse under a CC BY-NC-ND 3.0 licence after the 12 month embargo period.

After the embargo period, everyone is permitted to use copy and redistribute this article for non-commercial purposes only, provided that they adhere to all the terms of the licence https://creativecommons.org/licences/by-nc-nd/3.0

Although reasonable endeavours have been taken to obtain all necessary permissions from third parties to include their copyrighted content within this article, their full citation and copyright line may not be present in this Accepted Manuscript version. Before using any content from this article, please refer to the Version of Record on IOPscience once published for full citation and copyright details, as permissions will likely be required. All third party content is fully copyright protected, unless specifically stated otherwise in the figure caption in the Version of Record.

View the article online for updates and enhancements. 


\title{
Exploring Event Horizons and Hawking Radiation through Deformed Graphene Membranes
}

Tommaso Morresi, ${ }^{1,2}$ Daniele Binosi, ${ }^{1}$ Stefano Simonucci, ${ }^{3}$ Riccardo Piergallini, ${ }^{3}$ Stephan Roche, ${ }^{4,5 *}$ Nicola M. Pugno, ${ }^{6,7}$ Simone Taioli ${ }^{1,8 *}$

${ }^{1}$ European Centre for Theoretical Studies in Nuclear Physics and Related Areas (ECT*-FBK), Trento, Italy

${ }^{2}$ Institut de Minéralogie, de Physique des Matériaux et de Cosmochimie, Université Pierre et Marie Curie, Case 115, 4 Place Jussieu, 75252 Paris Cedex 05, France ${ }^{3}$ School of Science and Technology, University of Camerino, Camerino, Italy

${ }^{4}$ Catalan Institute of Nanoscience and Nanotechnology (ICN2), CSIC and BIST, Campus UAB, Bellaterra, Barcelona, Spain

${ }^{5}$ ICREA, Institucio Catalana de Recerca i Estudis Avancats, Barcelona, Spain

${ }^{6}$ Laboratory of Bio-inspired, Bionic, Nano, Meta Materials \& Mechanics, Department of Civil, Environmental and Mechanical Engineering, University of Trento, Italy

${ }^{7}$ School of Engineering and Materials Science, Queen Mary University of London, UK

${ }^{8}$ Trento Institute for Fundamental Physics and Applications (TIFPA-INFN), Trento, Italy

E-mail: taioli@ectstar.eu, stephan.roche@icn2.cat

\begin{abstract}
Analogue gravitational systems are becoming an increasing popular way of studying the behaviour of quantum systems in curved spacetime. Setups based on ultracold quantum gases in particular, have been recently harnessed to explore the thermal nature of Hawking's and Unruh's radiation that was theoretically predicted almost 50 years ago. For solid state implementations, a promising system is graphene, in which a link between the Dirac-like low-energy electronic excitations and relativistic quantum field theories has been unveiled soon after its discovery. This link could be extended to the case of curved quantum field theory when the graphene sheet is shaped in a surface of constant negative curvature, known as Beltrami's pseudosphere. Here we provide numerical evidence that energetically stable negative curvature graphene surfaces can be realized. Owing to large-scale simulations, our geometrical rèalizations are characterised by a ratio between the carbon-carbon bond length and the pseudosphere radius small enough to allow the formation of an analog of a black hole event horizon. Additionally, from the energy dependence of the spatially resolved density of states, we infer some thermal properties of the corresponding gravitational system, which could be investigated using low temperature scanning tunnelling microscopy or optical near field spectroscopy. These findings pave the way to the realization of a solid-state system in which the curved spacetime dynamics of quantum many body systems can be investigated.
\end{abstract}

Quantum mechanics and general relativity are the most successful theories of 


\section{Exploring Horizons and Hawking Radiation through Graphene Membranes}

modern physics. Most of the predicted exotic phenomena, from the weirdness of quantum entanglement to the existence of black holes have been experimentally tested and verified. On the other hand, a serious difficulty remains to merge those two fundamental theories in a single framework, which, in turn, makes it extremely challenging to obtain firm theoretical predictions.

One remarkable exception is the discovery by Hawking that, from a quantum mechanical point of view, black holes are not completely black [1]: they emit 'Hawking radiation' consisting of photons, neutrinos and, to a lesser extent, all sorts of massive particles. However, direct detection of this radiation, which is thermal in nature, seems beyond the experimental reach: Hawking radiation is in fact predicted to be proportional to the inverse of the black hole mass, which, for the smallest observed black hole, implies $T=60$ nK, i.e., 9 orders of magnitude smaller than the current cosmic microwave background temperature.

On the other hand, so-called black hole analogues, first/proposed by Unruh [2], are rapidly turning from promising to consolidated avenues in the study of various thermodynamics aspects. This is particularly true for sonic analogues built from ultracold gases $[3,4,5,6,7,8,9,10,11,12]$, for which not only Unruh-[13] and Hawking-like [14] radiation has been experimentally observed, but, in the latter case, its correlation spectrum shown to be thermal and with a temperature given by the system's surface gravity [15], thus vindicating Hawking's predictions.

The state-of-the-art of solid-state black hole analogues is, on the other hand, at a less advanced stage $[16,17,18]$. Indeed, while all current experimental approaches face major challenges mainly related to material synthesis and device fabrication, in the last couple of years key conceptual advances have been achieved; thus, there are now hopes for some of the fundamental questions to be addressed in condensed matter systems too, especially in connection to the implementation of the Sachdev-Ye-Kitaev model [19, 20] and its potential to holographically realize quantum black holes.

Here we propose a possible realization of a solid-state black-hole analogue. This system consists of a graphene membrane characterized by a three-connected tessellation engineered to shape it in the form of a constant negative curvature surface, known as Beltrami's pseudosphere $[22,23,24]$. In particular, we develop a novel computational method to build realistic and energetically stable negative curvature carbon allotropes comprising millions of atoms. Furthermore, we elaborate a tight-binding (TB) approach to calculate the local density of state (LDOS) for these extended curved structures. We anticipate that, despite the comparison between the numerically evaluated and the theoretically predicted LDOSs is inherently plagued by the formation of pentagonal and heptagonal defects in our realistic structures, we find an asymmetric LDOS around the Fermi level, which has been predicted to encode the signature of gravitational horizon of events in black hole physics, through the thermal properties of related electronic excitations $[22,23]$.

Beltrami's pseudosphere represents the hyperbolic counterpart of the regular sphere: it is a surface of revolution characterized by a constant negative Gaussian curvature 

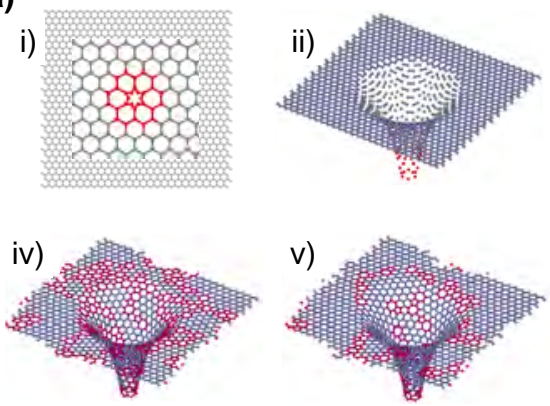

Exploring Horizons and Hawking Radiation through Graphene Membranes
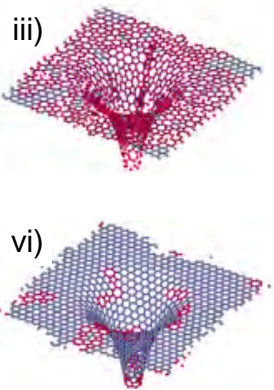

b)

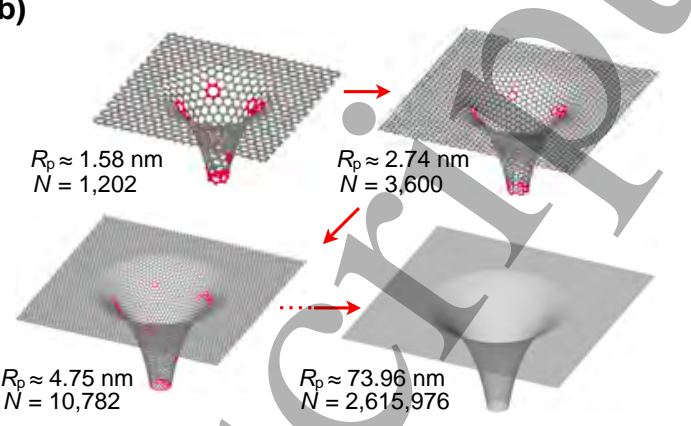

Figure 1: Tiling the hyperbolic plane by three-coordinated tessellations realized by an all- $s p^{2}$ carbon-based net. a) Optimization of a carbon pseudosphere containing $N=1626$ atoms with radius $R_{\mathrm{p}}=18.26 \AA$. Panels from i to vil represent different optimization stages, consisting of trial bond switch-twist moves. Carbon atoms that do not belong to the hexagonal faces are marked in red color. Starting from an initial configuration (panel i) almost entirely tiled with hexagonal polygons, atoms rearrange to fill uniformly the surface (panels ii to v) reaching a local mimimum after a few thousands steps (panel vi). After reaching this configuration, trial moves are rejected at an increasingly high rate and the Metropolis algorithm becomes inefficient. b) A dualization sequence (described in Supplemental Material [21]), leading to a thousandfold increase in the number of carbon atoms and close to a hundredfold increase in the pseudosphere radius.

$\kappa=-1 / R_{\mathrm{p}}^{2}$, with $R_{\mathrm{p}}$ the pseudosphere radius.

Under suitable boundary conditions, Gauss Bonnet's theorem shows that the existence of Stone-Wales (SW) defects with an excess of six heptagonal defects with respect to the pentagonal units $[24,25,26]$ is required to tile the pseudosphere with carbon atoms. Thus, the presence of six heptagonal shapes is imposed at the beginning and preserved by all the steps of the construction. In addition, Hilbert's theorem states that no analytic complete surfaces of constant negative Gaussian curvature can be embedded in $\mathbb{R}^{3}$, implying that the graphene pseudosphere cannot be complete.

Early investigations to build a realistic Beltrami's pseudosphere by finding a (local) minimum energy tiling of carbon atoms taking into account these two theorems [24], have been inconclusive in: i) delivering a general approach to the tessellation of hyperbolic surfaces; ii) scaling-up the graphene pseudosphere size; and iii) measuring the surface's electronic structure. And properties ii) and iii) are of paramount importance in aseertaining the capacity of this carbon-based structure to act as an analogue gravity model.

Our method proceeds as follows: we start the pseudosphere generation from a planar graphene sheet, in which we impose the presence of six heptagonal faces in the center (see Fig. 1a-i). The initial configuration of the pseudosphere (Fig. 1a-ii) is then obtained by simply projecting the graphene net on the Beltrami's surface along the $z$-axis (see Supplemental Material [21]). In this configuration, the carbon-to-carbon 


\section{Exploring Horizons and Hawking Radiation through Graphene Membranes}

bond lengths in the bent region within the pseudosphere is longer than the typical bond distances in flat graphene $\left(a_{\mathrm{CC}}=1.42 \AA\right)$, owing to the (negative) curvature. Next, a sequence of bond-switching trial moves and structural optimization steps with a modified Keating potential to favour the formation of hexagonal cells is then applied (see Supplemental Material [21], Fig. 5a), and accepted or rejected according to a suitable energy minimization criterion (Fig. 1a, panels iii through v). After $\mathcal{O}\left(10^{4}\right)$ moves the algorithm efficiency drastically drops, which limits the radius size of the minimized structures (Fig. 1a vi) to few nm and the number of carbon atoms to $\mathcal{O}\left(10^{3}\right)$.

Scaling-up of the numbers of atoms to achieve satisfactory experimental conditions (which will be discussed below) is next implemented through a custom dualization algorithm (Figs. 1b and 5b), by which the pseudosphere radius and number of atoms scale like $\sim \sqrt{3}$ and $\sim 3$ respectively, while conserving both the bond distance as well as the number of defects (see Supplemental Material [21]). Each dualization step is then followed by a bond switching optimization run to counteract the former tendency of splitting apart the SW defects of the original structure (and, thus, artificially increasing its total energy). Repeated application of this procedure allows one to reach a thousandfold increase in the number of carbon atoms (our maximum value being $N=2,615,976$ ) and a pseudosphere radius $R_{\mathrm{p}}=73.96 \mathrm{~nm}$. We hasten to emphasize that these atomic configurations are found to be stable also by molecular dynamics simulations at several thousands K. More specifically, despite the formation of ripples and local deformations in proximity of the defected sites, graphene membranes of minimal energy result dynamically stable also when relaxing the condition that carbon atoms are strictly located on the analytical Beltrami's surface.

The signature of the Hawking-Unruh effect in the carbon pseudosphere can be found by characterizing the electronic properties in terms of the LDOS near the Dirac points [27], where electrons behave as relativistic massless pseudo-particles. Given the $\mathcal{O}\left(10^{6}\right)$ carbon atoms of the realized structures, the LDOS will be evaluated through a multi-orbital TB approach implementing the Kernel Polynomial Method (KPM) to avoid the diagonalization of the Hamiltonian [28]. Due to curvature, in fact, the $p_{z}$ orbitals contributing to the $\pi$ band are not anymore orthogonal to the in-plane direction; similarly, the $s p^{2}$-hybridized orbitals do not lay in the graphene plane. Thus, an approach, in which all four valence orbitals $\left(2 s, 2 p_{x}, 2 p_{y}, 2 p_{z}\right)$ are included in the simulations as opposed to the $p_{z}$ orbital alone, has been necessary (see Supplemental Material[21] and Fig. 6 therein, where details concerning the parametrization of the Hamiltonian are reported and well-established results on graphene and carbon nanotube structures reproduced).

The LDOS projected onto longitudinal circles in regions located at a different $z$ depth along pseudospheres obtained at various stages of the dualization procedure (and thus characterized by varying number of atoms $N$ and radius $R_{\mathrm{p}}$ ), is plotted in Fig. 2 . In each case we evaluate this quantity for three structures differing by the number and location of the SW defects. In the energy range $E \in[-6,6] \mathrm{eV}$, the LDOS shows a graphene-like shape for all the pseudospheres independently of the radius and defect 
a)
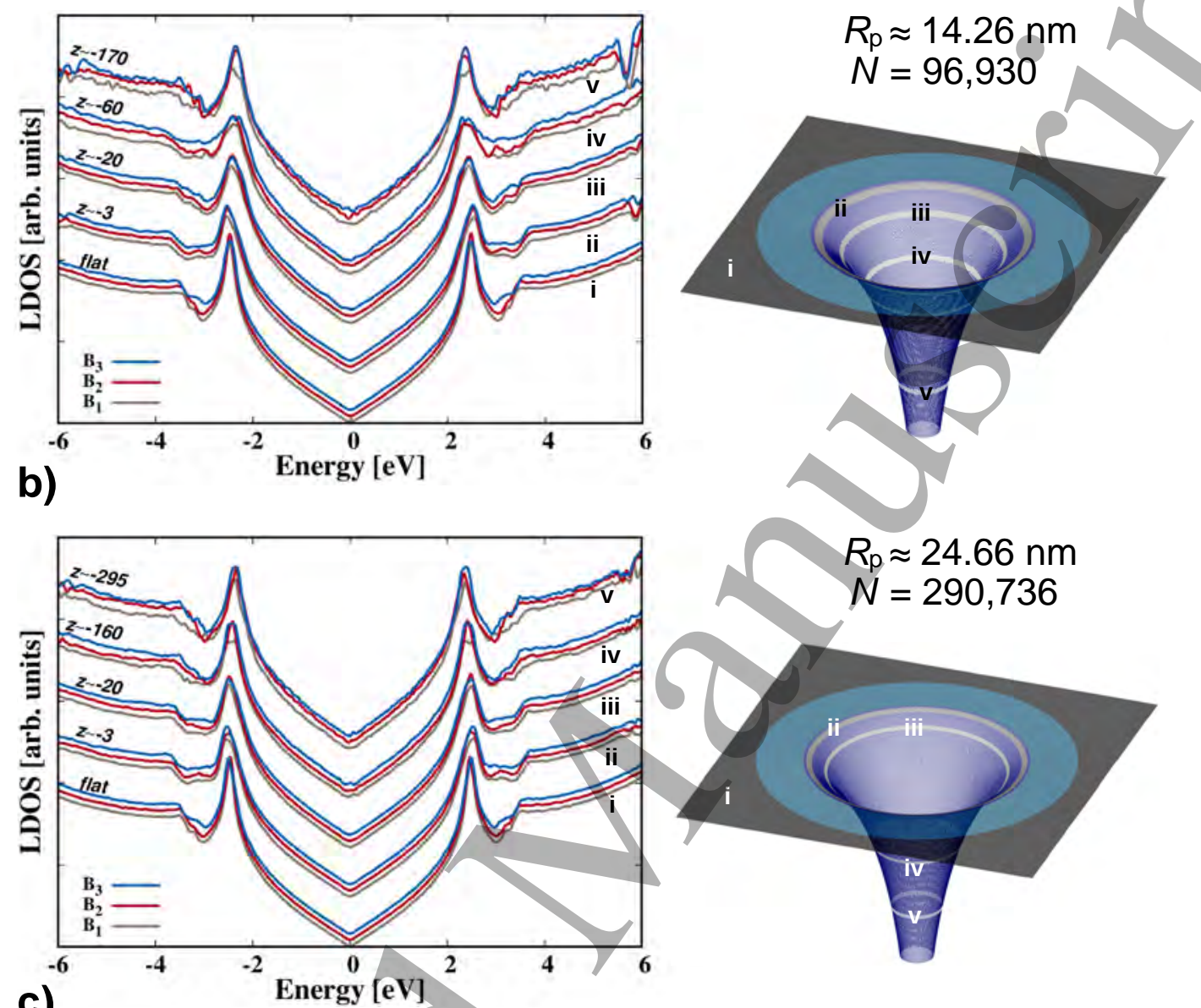

c)
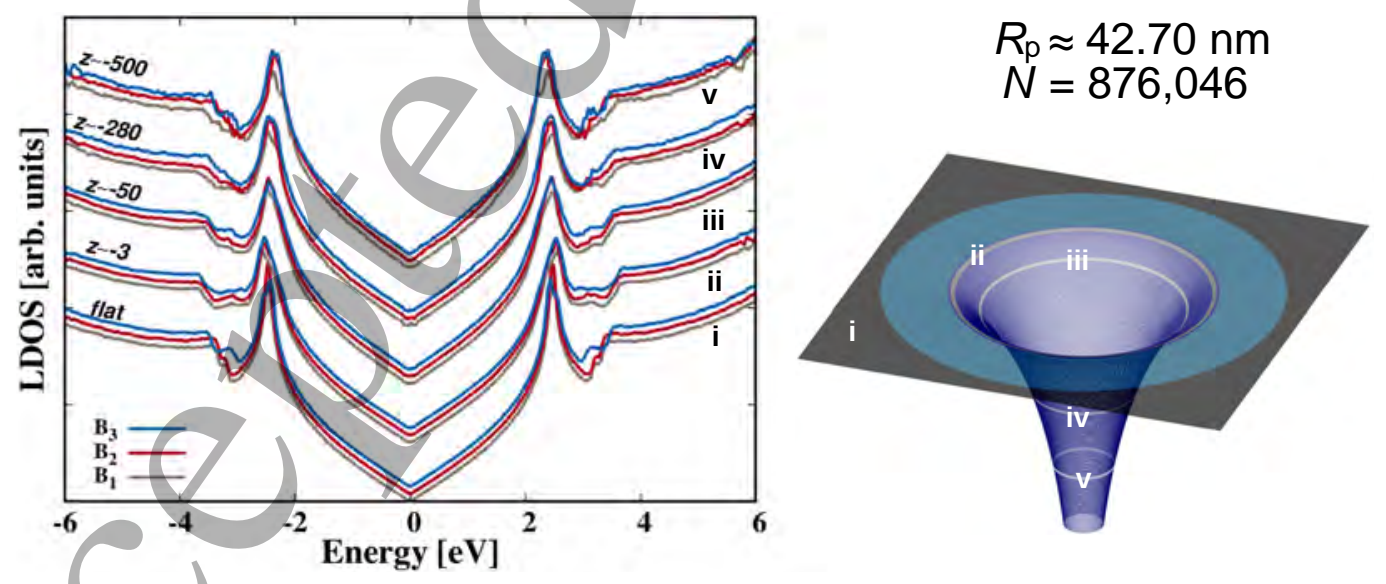

Figure 2: Evaluation of the LDOS through a multi-orbital TB approach implementing the Kernel Polynomial Method. Panels a) through c): LDOS projected onto five regions at different depth along the revolution axis $z$ (i through $\mathrm{v}$ ) for various pseudospheres characterized by three different values of the number of atoms $N$ and radius $R_{\mathrm{p}}$. For each case of $N$ and $R_{\mathrm{p}}$ we report the LDOS for three pseudosphere realizations $\mathrm{B}_{1-3}$ differing by the configurations of SW defects, of which a representative is shown on the right of each panel. The Fermi energy is set equal to zero in all cases. 
Exploring Horizons and Hawking Radiation through Graphene Membranes
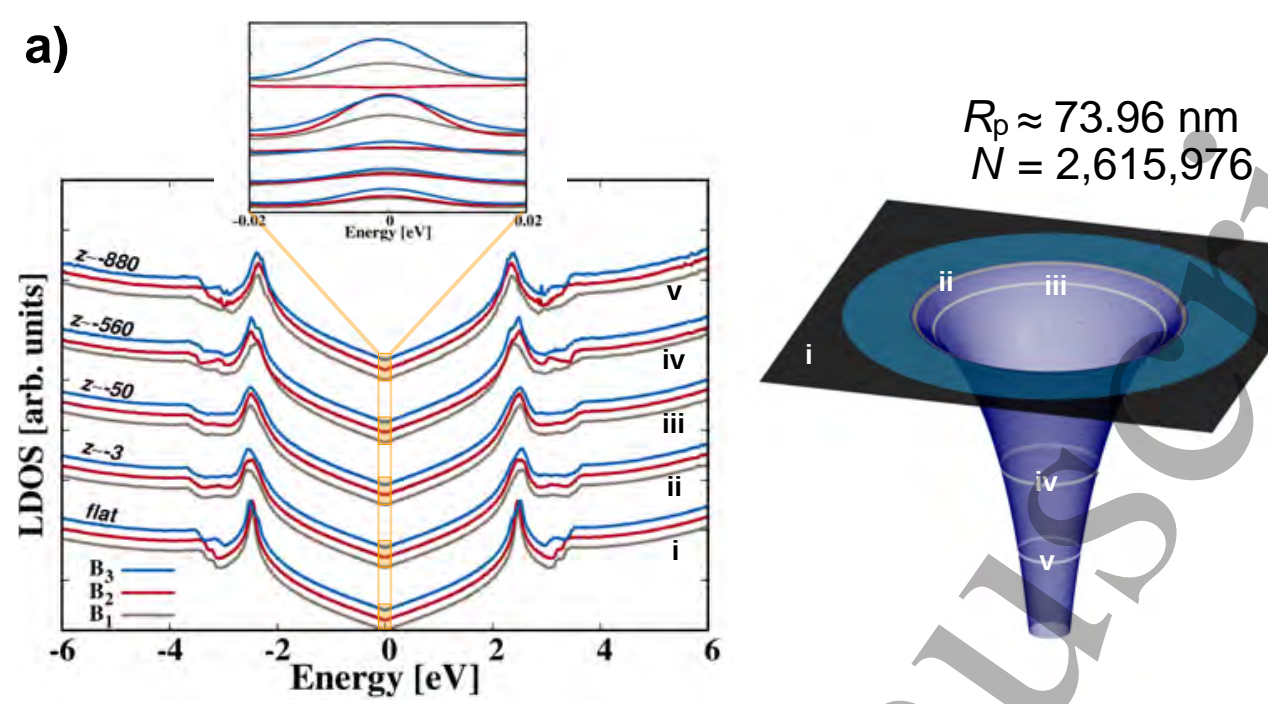

b)
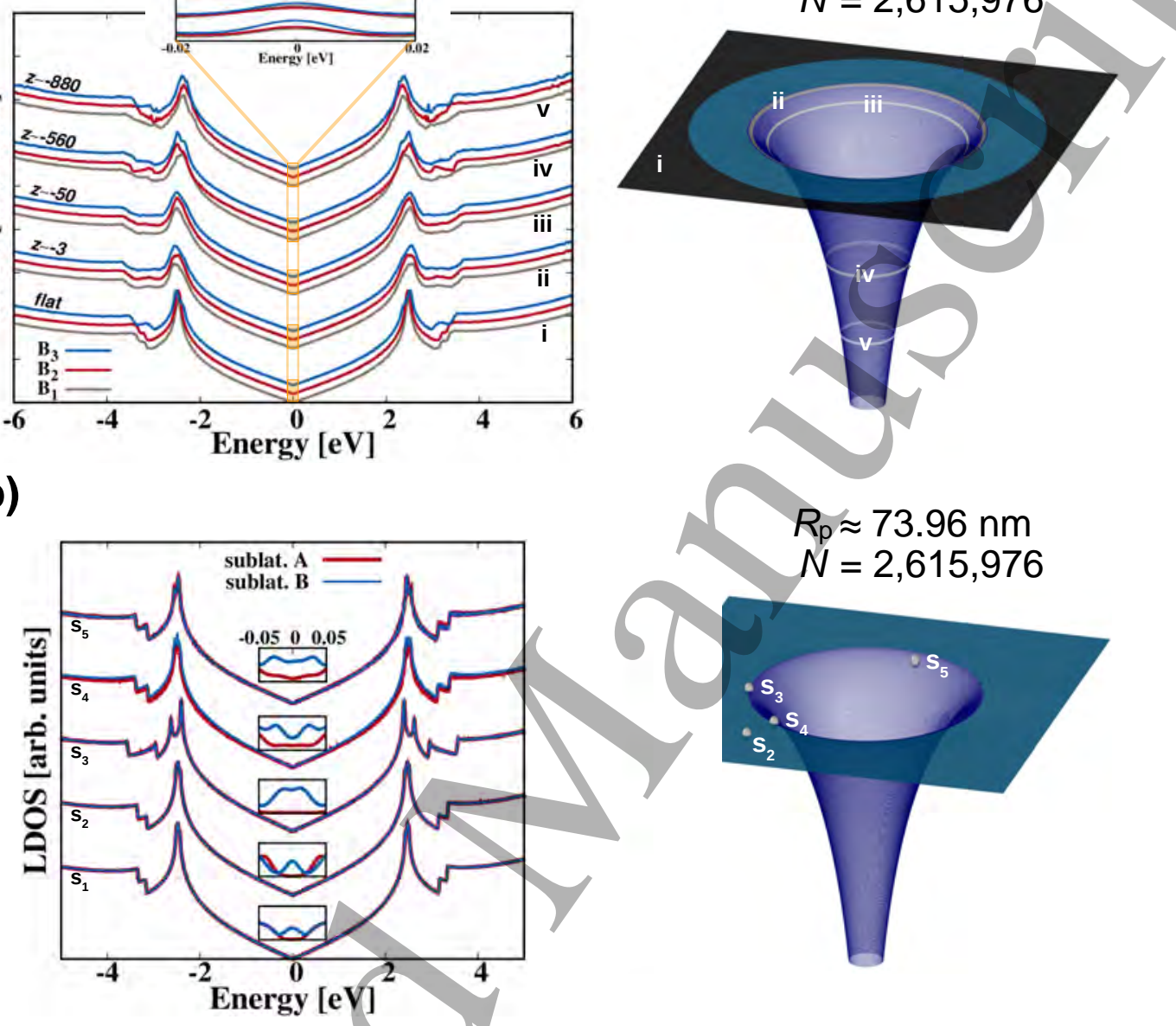

Figure 3: LDOS symmetry breaking due to curvature effetcs. a) LDOS projected onto five regions at different depth along the revolution axis $z$ (i through $\mathrm{v}$ ) for for the three biggest pseudospheres ( $N=2,615,976$ and $R_{\mathrm{p}}=73.96 \mathrm{~nm}$, right). We also zoom on the Fermi energy to expose the LDOS bulge in this region. b) Pseudosphere's LDOS projected over the shown sites $s_{i}$. Projection over the two inequivalent sublattices A and $\mathrm{B}$ of graphene is also shown; the insets zoom near the Fermi energy to show the LDOS asymmetry.

distribution. With respect to the pristine graphene (region i), region ii shows VanHove singularities associated to the $\pi$ band peaks which are broadened and shifted; this is due to the slightly elongated carbon bonds characterizing this pseudosphere region, which represents the would-be Hilbert horizon (where the pseudosphere ends as a consequence of the Hilbert theorem). Moving further inside, the LDOS stays the same at a qualitatively level independently of the pseudosphere funnel depth at which is evaluated.

A similar overall behaviour (Fig. 3a) persists in the biggest structures studied. However, by zooming in the vicinity of the Fermi energy we find a bulge, which can be 
a)

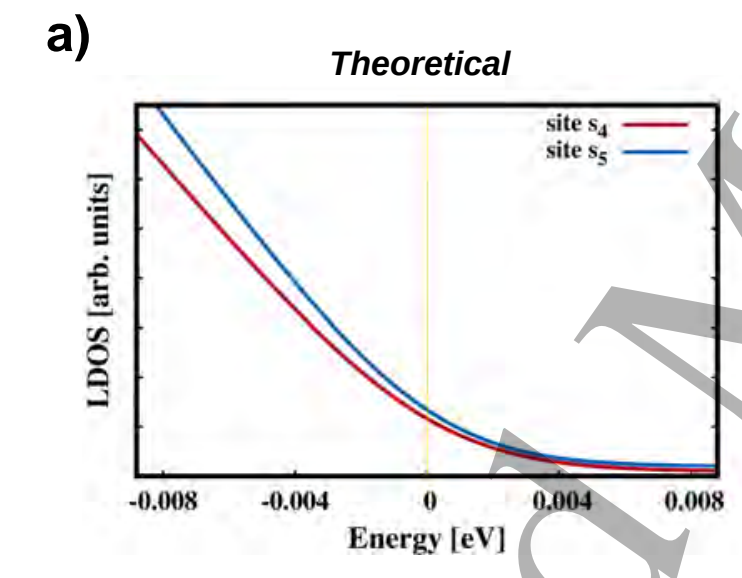

\section{Exploring Horizons and Hawking Radiation through Graphene Membranes}

seen in the blown up region in Fig. 3a) and which could be also spotted in the central region of Fig. 2. We attribute this behavior of the LDOS to a genuine curvature effect. Projecting the LDOS over single atomic sites both inside and outside the pseudosphere (sites $s_{1-5}$ in Fig. $3 \mathrm{~b}$ ) and disentangling the nearest-neighbour contributions (that would correspond to the $\mathrm{A}$ and $\mathrm{B}$ sublattices in pristine graphene), we find that the LDOS spectrum around the Fermi energy $E_{\mathrm{F}}$ is significantly asymmetric for the two nonequivalent sublattices, while for energy $E \gg E_{\mathrm{F}}$ it is practically indistinguishable. It is worth to notice that axial symmetry of our realistic structures is broken by the presence of the defects, at variance with the continuum mathematical model where one deals with a revolution surface. Furthermore, we stress that this finding agrees with a similar behavior in strained graphene sheets [29], where sublattice symmetry breaking was induced by an out-of-plane deformation. Further details on LDOS calculations and Beltrami's geometry generation can be found in the Supplemental Material.

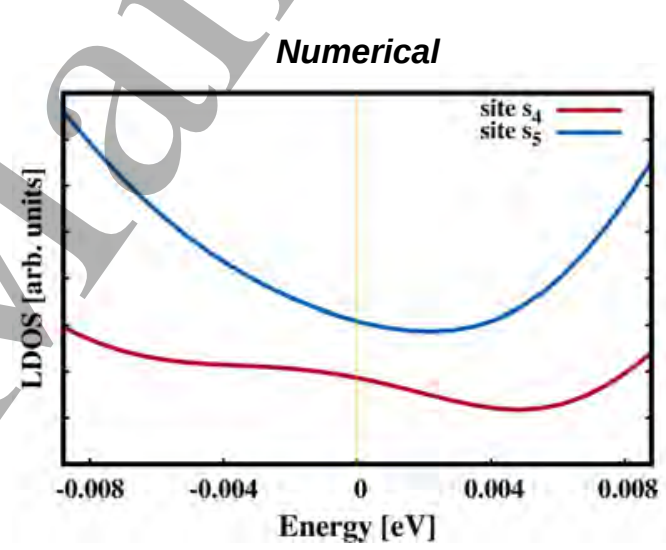

b)
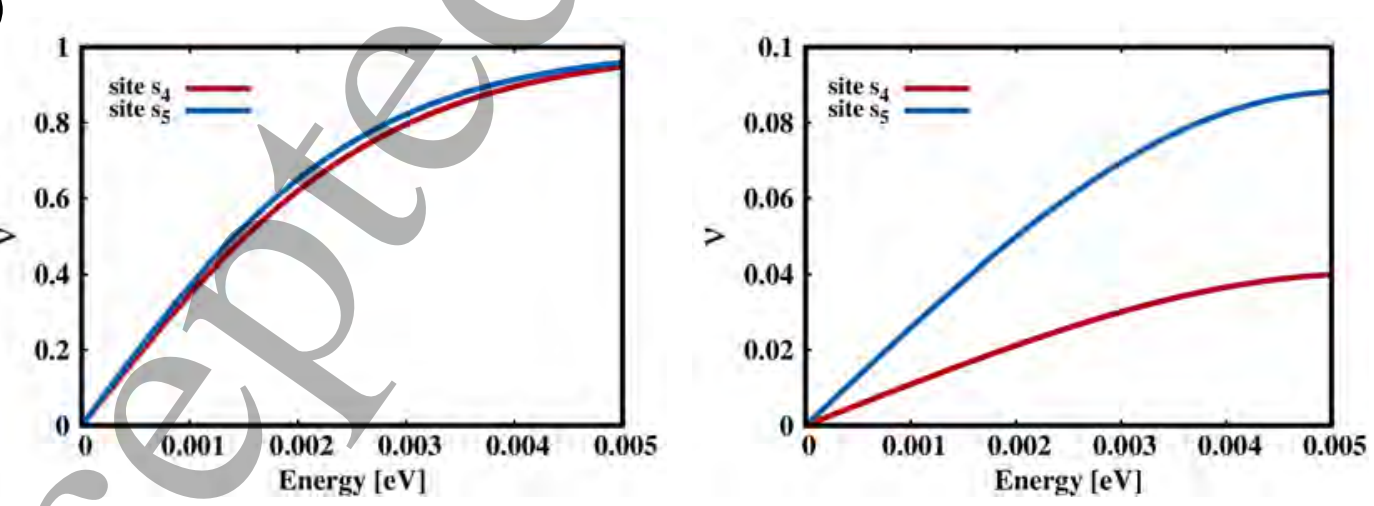

Figure 4: Detecting the presence of an event horizon through the low-energy LDOS. LDOS lineshape (a) and contrast (b) projected over the pseudosphere sites $s_{4,5}$ shown in Fig. 3b), according to analytical predictions [22, 23] (left) and numerical results (right).

The largest simulated pseudosphere has a ratio $a_{\mathrm{CC}} / R_{\mathrm{p}} \sim 2 \times 10^{-3}$. This parameter determines how well the Hilbert horizon and the Rindler-type event horizon, emerging when treating the pseudosphere as a $2+1$ dimensional space-time in which the valence 


\section{Exploring Horizons and Hawking Radiation through Graphene Membranes}

electrons move, coincide $[22,23]$ (0 representing coincidence). In the ideal case [22, 23], in the proximity of the horizon, Hawking's radiation happens due to massless electrons, which before tunnelling (i.e. on the pseudosphere surface) are described by the action

$$
S=i v_{\mathrm{F}} \int \mathrm{d}^{3} x \sqrt{g} \bar{\psi} \gamma^{\mu} \mathcal{D}_{\mu} \psi
$$

where $v_{\mathrm{F}} \sim c / 300$ is the Fermi velocity, $\gamma^{\mu}$ are the Dirac matrices, $\psi$ and $\bar{\psi}$ are the field operators creating particles and holes respectively, and $\mathcal{D}_{\mu}$ is the $\operatorname{SO}(2,1)$ covariant derivative. Finally, $g$ is the determinant of the pseudosphere metric $\mathrm{d} s_{\mathrm{B}}^{2}=\phi^{2}(u) \mathrm{d} s_{\mathrm{R}}^{2}$, where $\phi(u)=\ell / r \mathrm{e}^{u / r}, \ell$ is a constant that in the physical case is to be identified with $a_{\mathrm{CC}}$ ) and $\mathrm{d} s_{\mathrm{R}}^{2}$ is the Rindler-type metric $\mathrm{d} s_{\mathrm{R}}^{2}=\phi^{-2}(u)\left(\mathrm{d} t^{2}-\mathrm{d} u^{2}\right)-r^{2} \mathrm{~d} v^{2}$ (with $u$ and $v$ the curvilinear coordinates spanning the pseudosphere). We stress at this point that Eq. (1) is strictly valid only at very small energies around the Fermi level and that the covariant derivative $\mathcal{D}_{\mu}$ does not take into account elastic effects for which the associated stress tensor would mix the four-component pseudo-spinor arising from the symmetry of the underlying honeycomb graphene lattice. After tunneling, the electrons move in a flat metric (the graphene plane) where the action is given by Eq. (1), with the replacements $\mathcal{D} \rightarrow \partial$, and $g \rightarrow 1$. The presence of an event horizon, can be revealed by evaluating the power spectrum $\rho$ of the 2-point function $S_{\mathrm{B}}=\left\langle 0_{\mathrm{M}}\left|\psi_{\mathrm{B}} \bar{\psi}_{\mathrm{B}}\right| 0_{\mathrm{M}}\right\rangle$, being $|0\rangle_{\mathrm{M}}$ the flat vacuum; in this case, it would assume a thermal form $[22,23]$ :

$$
\rho=\frac{4}{\pi} \frac{1}{\left(\hbar v_{\mathrm{F}}\right)^{2}} \frac{R_{\mathrm{p}}^{2}}{a_{\mathrm{CC}}^{2}} \mathrm{e}^{-2 u / R_{\mathrm{p}}} \frac{E}{\mathrm{e}^{\frac{E}{k_{\mathrm{B}}}}-1} ; \quad \Theta=\frac{\hbar v_{\mathrm{F}}}{k_{\mathrm{B}}} \frac{a_{\mathrm{CC}}}{2 \pi R_{\mathrm{p}}^{2}} \mathrm{e}^{u / R_{\mathrm{p}}},
$$

where $k_{\mathrm{B}}$ is the Boltzmann constant and $\Theta$ the temperature. At the horizon, where $u=$ $R_{\mathrm{p}} \ln R_{\mathrm{p}} / a_{\mathrm{CC}}$, the Hawking temperature reaches its maximum $\Theta=\hbar v_{\mathrm{F}} /\left(2 \pi k_{\mathrm{B}} R_{\mathrm{p}}\right) \sim 16$ $\mathrm{K}$ for our largest pseudosphere. Notice that this is a low energy effect: only electrons with an intrinsic energy $E=\hbar v_{\mathrm{F}} / R_{\mathrm{p}} \sim 9 \mathrm{meV}$ have a wavelength long enough to experience the effects of the curvatures and thus a LDOS described by (2). Furthermore, the large radius requirement implicit in the intrinsic energy scale emerging from the graphene pseudosphere analog model rigorously justifies the low-energy description in terms of massless electron and holes. On the other hand, for detecting experimentally the Hawking temperature associated to the existence of the Rindler horizon, $R_{\mathrm{p}}$ should be not extremely large, as Éq. (2) implies $\Theta \propto 1 / R_{p}$. Thus, the optimal radius value turns out to be a trade-off between these two opposite requirements. We notice that already for $a_{\mathrm{CC}} / R_{\mathrm{p}} \sim 10^{-2}$ (see Fig. 2) the approximation of the Rindler event horizon with the Hilbert horizon of the Beltrami's spacetime is rather accurate and the LDOS asymmetry is emerging. Nevertheless a pseudosphere with a radius in the range of $\mu \mathrm{m}$ is necessary to achieve a good resolution in the linear part of spectrum.

Given the exponential nature of the power spectrum (2), the effect of the presence of the horizon should manifest in a marked asymmetry of the LDOS around the Fermi energy, as measured by the contrast

$$
\nu(E)=\left|\frac{\operatorname{LDOS}(-E)-\operatorname{LDOS}(E)}{\operatorname{LDOS}(-E)+\operatorname{LDOS}(E)}\right| .
$$


Results for the LDOS and its contrast projected on sites located near the Hilbert/Rindler horizon are shown in Fig. 4 for the theoretical (left) and numerical (right) predictions. Here we stress two points: first, that the continuum model approximation disregards the impact of atomistic defects and their consequence on emerging strain fields on the LDOS, and thus on Eq. (2) predictions. At variance, the Gauss-Bonnet theorem links indissolubly defects and curvature as two different aspects of the same effect. To disentangle the impact of curvature and defects on the LDOS, calculations for planar graphene including a random density of SW defects were performed (see Supplemental Material). In this case, the LDOS loses its symmetric behaviour in the linear part of the spectrum around the Fermi energy more evidently close to the defective sites. Furthermore, the presence of elastic strain also generates an asymmetric splitting of the LDOS of the two sublattices A and B near the charge neutrality point (see Figs. 3a and 3b) [29]. In a realistic model of Beltrami's pseudosphere the fabricated structures are open at the bottom, where the continuum analytical model has a singularity. In this regard, we notice that Eq. (2) neglects boundary effects, which appear owing to the finite size of the Beltrami's pseudosphere in real world experiments. Static boundaries, such as the pseudosphere truncation to avoid carbon atoms unphysically compressed at the bottom singular point, enhance the oscillating behaviour of the LDOS around the Fermi level by including non-thermal features that smear the QFT approach in curved spacetime (see Eq. (95) of Ref. [23] for more details on boundary terms). We also stress that the assessment of the DOS in hyperbolic spaces has been tempted by other authors (see e.g. [30]).

We notice that the assessment of the DOS in hyperbolic spaces has been tempted by other authors (see e.g. Ref. [30]). We stress that a Lobachevsky plane represents an hyperbolic structure only locally (but not globally) isometric to the Beltrami's pseudosphere. Thus, the solution of global integral equations can differ. Furthermore, the emergence of an horizon and of the Hawking temperature therein cannot be captured in a simple hyperbolic space. Nevertheless, we also point out that Eq. (2) describes the same low-energy limit $(E \rightarrow 0)$ around the Fermi energy of the DOS in flat euclidean spaces $(\rho(E) \simeq|E|))$ as well as the same finite value of the DOS at $E=0$ previously found (see Eq. (19) of Ref. [30]). However, a central feature of the LDOS of realistic carbon pseudospheres is to show asymmetric behaviour.

The quantitative discrepancy found between the purely theoretical predictions and our numerical results is explained by these differences in the modelling, which have a significant impact on the assessment of the electronic properties of the pseudosphere. Despite this, the similar qualitative agreement is key to confirm that the more realistic atomistic modelling of graphene pseudospheres retains essential features describing the low energy electronic properties, which are driving the analogy to black holes thermodynamics.

In conclusion, we have designed a realistic model of a Beltrami's pseudosphere tiled by carbon atoms arranged in a defected graphene net. The latter is found to be an energetically and dynamically stable allotrope of carbon. Its corresponding LDOS, 


\section{Exploring Horizons and Hawking Radiation through Graphene Membranes}

obtained numerically by solving a tight-binding Hamiltonian, reproduces qualitatively the analytical model, where an asymmetric behaviour is predicted. However, in order to consider undoubtedly the Beltrami's pseudosphere tiled by graphene-a viable solid state analogue of a quantum field theory in curved space-time in general, and a blackhole horizon in particular, we believe that significant theoretical advances, based also on our computational findings, should point towards the assessment of the decisive impact that the presence of defects has on the intertwining of the $A$ and $B$ sublattice contribution to the Dirac pseudo-spinors in relation to Eq. (1). Indeed, we find a striking evidence that this effect cannot be neglected as done so far in analytical models. In fact the latter theoretical efforts recognized that massless electron-hole pair generation at the Hilbert horizon of the graphene pseudosphere as measured by the LDOS is analogous to Hawking radiation in conventional black holes; but while in those systems the radiation temperature is too small to be observed directly, in the carbon pseudosphere temperatures of the order of tens of $\mathrm{K}$ are in principle attainable. We stress that the success of our numerical computations to generate a realistic discrete model for testing the analytical predictions obtained within a continuum representation paves the way for a deeper understanding of solid-state black-hole analogues as well as makes it possible to design a suitable experimental platform for further investigations.

In particular, from the experimental point of view, the manufacturing of a carbon pseudosphere can be achieved via optical forging of graphene on a copper substrate. This nanofabrication technique produces ripples by inducing defects, such as StoneWales chains, which cause strain and, thus, bending of the graphene sheet with features unlimited by diffraction (see Ref. [31]). The defect pattern reflects the light intensity Gaussian distribution of the focused laser spot. In particular, one can envisage to shape graphene into a specific curvature by following two different strategies: either by modulating the intensity distribution within the laser spot, or by using tip-enhanced optical forging to locate defects with 10-20 nm accuracy. Strain would be induced point-by-point until the desired shape, originating by the interplay with the substrate adhesion, is created. Moreover, to forge the Beltrami's structure one needs to start from an open graphene sheet, in which the central hole can be milled by a helium ion microscope [31] or by tip-enhanced ablation of graphene. The optical forging technique thus offers an accurate way to modify and shape 2D materials and facilitates the creation of controllable nanostructures. Additionally, the thermal character of the low energy LDOS can be ascertained through either low temperature scanning tunnelling microscopy or optical near-field spectroscopy.

\section{Acknowledgments}

S.T. acknowledges discussion with Andreas Johansson (University of Jyväskylä, Finland) for the experimental realization of the carbon pseudosphere by optical forging of graphene. ICN2 is funded by the CERCA Programme/Generalitat de Catalunya, and is supported by the Severo Ochoa program from Spanish MINECO 


\section{References}

[1] Hawking S W 1974 Nature 248 30-31

[2] Unruh W G 1981 Phys. Rev. Lett. 46 1351-1353

[3] Jacobson T A and Volovik G E 1998 Phys. Rev. D58 064021 (Preprint cond-mat/9801308)

[4] Garay L J, Anglin J R, Cirac J I and Zoller P 2000 Phys. Rev. Lett. 85 4643-4647 (Preprint gr-qc/0002015)

[5] Barcelo C, Liberati S and Visser M 2003 Int. J. Mod. Phys. A18 3735 (Preprint gr-qc/0110036)

[6] Giovanazzi S 2005 Phys. Rev. Lett. 94061302

[7] Balbinot R, Fabbri A, Fagnocchi S, Recati A and Carusotto I 2008 Phys. Rev. A78 021603 (Preprint 0711.4520)

[8] Carusotto I, Fagnocchi S, Recati A, Balbinot R and Fabbri A 2008 New J. Phys. 10103001 (Preprint 0803.0507)

[9] Macher J and Parentani R 2009 Phys. Rev. A80 043601(Preprint 0905.3634)

[10] Recati A P N and Carusotto I 2009 Phys. Rev. A80 043603 (Preprint 0907.4305)

[11] Larre P E, Recati A, Carusotto I and Pavloff N 2012 Phys. Rev. A85 013621 (Preprint 1110.4464)

[12] Steinhauer J 2015 Phys. Rev. D92 024043 (Preprint 1504.06583)

[13] Hu J, Feng L, Zhang Z and Chin C 2019 Nature Phys. S41567 0537-1 (Preprint 1807.07504)

[14] Steinhauer J 2016 Nature Phys. 12959 (Preprint 1510.00621)

[15] de Nova J R M, Golubkov K, Kolobov V I and Steinhauer J 2019 Nature 569 688-691 (Preprint 1809.00913)

[16] Franz M and Rozali M 2018 Nature Rev. Mater. 3 491-501 (Preprint 1808.00541)

[17] Cvetic M and Gibbons G 2012 Annals of Physics 3272617 - 2626 ISSN 0003-4916 URL http://www.sciencedirect.com/science/article/pii/S0003491612000814

[18] Gonzalez J and Herréro J 2010 Nuclear Physics B 825426 - 443 ISSN 0550-3213 URL http://www.sciencedirect.com/science/article/pii/S0550321309005112

[19] Sachdev S and Ye J 1993 Phys. Rev. Lett. 703339 (Preprint cond-mat/9212030)

[20] Kitaev A KITP Strings Seminar and Entanglement Program

[21] See Supplemental Materia1, which includes Refs. [27-36], for FIGS. 5 and 6 and for further information on: (i) Tiling the pseudosphere; (ii) Tight-binding parameter estimate; (iii) Kernel Polynomial Method; (iv) Tests of the LDOS calculations; (v) LDOS of a single SW defect in planar graphene.

[22] Iorio A and Lambiase G 2012 Phys. Lett. B 716 334-337 (Preprint 1108.2340)

[23] Iorio A and Lambiase G 2014 Phys. Rev. D 90025006 (Preprint 1308.0265)

[24] Taioli S, Gabbrielli R, Simonucci S, Pugno N M and Iorio A 2016 Journal of Physics: Condensed Mattèr 28 13LT01 URL http://stacks.iop.org/0953-8984/28/i=13/a=13LT01

[25] Tatti R, Aversa L, Verucchi R, Cavaliere E, Garberoglio G, Pugno N M, Speranza G and Taioli S 2016 RSC Advances 6 37982-37993

[26] Taioli S 2014 Journal of molecular modeling 202260

[27] Castro Neto A H, Guinea F, Peres N M R, Novoselov K S and Geim A K 2009 Rev. Mod. Phys. 81(1) 109-162 URL https://link.aps.org/doi/10.1103/RevModPhys.81.109

[28] Torres L, Roche S and Charlier J 2014 Introduction to Graphene-Based Nanomaterials: From 
Exploring Horizons and Hawking Radiation through Graphene Membranes

Electronic Structure to Quantum Transport (Cambridge University Press) ISBN 9781107655959 URL https://books.google.it/books?id=bbGkAgAAQBAJ

[29] Schneider M, Faria D, Viola Kusminskiy S and Sandler N 2015 Phys. Rev. B 91(16) 161407 URL https://link.aps.org/doi/10.1103/PhysRevB.91.161407

[30] Gorbar E and Gusynin V 2008 Annals of Physics 3232132 - 2146 ISSN 0003-4916 URL http://www.sciencedirect.com/science/article/pii/S0003491607001728

[31] Koskinen P and et al 2018 J. Phys. Chem. Lett. 9 6179-6184

[32] Kumar A, Wilson M and Thorpe M F 2012 Journal of Physics: Condensed Matter 24485003 URL http: //stacks.iop.org/0953-8984/24/i=48/a=485003

[33] Wooten F, Winer K and Weaire D 1985 Phys. Rev. Lett. 54(13) 1392-1395 URL https://link.aps.org/doi/10.1103/PhysRevLett.54.1392

[34] Bitzek E, Koskinen P, Gähler F, Moseler M and Gumbsch P 2006 Phys. Rev. Lett. 97(17) 170201 URL https://link.aps.org/doi/10.1103/PhysRevLett.97.170201

[35] Gould H, Tobochnik J and Christian W 1988

[36] Alfthan S 2006 Computational studies of silicon interfaces and amorphous silica G5 artikkeliväitöskirja URL http://urn.fi/urn:nbn:fi:tkk-008820

[37] Slater J C and Koster G F 1954 Phys. Rev. 94(6) 1498-1524 URL https://link.aps.org/doi/10.1103/PhysRev.94.1498

[38] Yuan S, Rösner M, Schulz A, Wehling T O and Katsnelson M I 2015 Phys. Rev. Lett. 114(4) 047403 URL https://link.aps.org/doi/10.1103/PhysRevLett.114.047403

[39] Stauber T, Beltran J I and Schliemann J 2016 Scientific Reports 622672 URL https://doi.org/10.1038/srep22672

[40] Giannozzi P, Baroni S, Bonini N, Calandra M, Car R, Cavazzoni C, Ceresoli D, Chiarotti G L, Cococcioni M, Dabo I, Corso A D, de Gironcoli S, Fabris S, Fratesi G, Gebauer R, Gerstmann U, Gougoussis C, Kokalj A, Lazzeri M, Martin-Samos L, Marzari N, Mauri F, Mazzarello R, Paolini S, Pasquarello A, Paulatto L, Sbraccia C, Scandolo S, Sclauzero G, Seitsonen A P, Smogunov A, Umari P and Wentzcovitch/R M 2009 Journal of Physics: Condensed Matter 21395502 URL http: //stacks.iop.org/0953-8984/21/i=39/a=395502

[41] Weiße A, Wellein G, Alvermann A and Fehske H 2006 Rev. Mod. Phys. 78(1) 275-306 URL https ://link.aps.org/doi/10.1103/RevModPhys.78.275

[42] Garcia J 2015 The kernel polynomial method for quantum transport in disordered systems Ph.D. thesis 


\section{Appendix}

\section{Tiling the pseudosphere}

The tiling of the Beltrami's pseudosphere by carbon atoms, which represents, an interesting geometrical problem in its own right, has been achieved through the following steps:

(i) Set the length of the pseudosphere by fixing the maximum value of the coordinate along the axis of revolution $(z)$.

(ii) Determine the number of carbon atoms $N$ that are needed if one were to tile the surface of the Beltrami's pseudosphere with the same density of planar graphene (0.379 atoms $\left./ \AA^{2}\right)$. Periodic boundary conditions are applied by using a rectangular supercell repeated along the $x$ and $y$ directions to saturate the outer carbon atom bonds belonging to $r=R_{\mathrm{p}}$ and $z=0$ (the Hilbert horizon).

(iii) Construct a planar graph $(N, F, E)$ consisting of $N$ vertices, $F$ faces and $E$ edges. The $N$ vertices represent compressed carbon atoms with shortened carbon-tocarbon bond lengths, $a_{\mathrm{CC}}<1.42 \AA$; each vertex is linked to three nearest neighbours by edges (representing bonds) and is shared by three faces.

(iv) Map the initial graph onto the Beltrami's pseudosphere surface via a one-toone transformation by which the revolution axis coordinate $z$ of the vertices is unambiguously determined $\forall \sqrt{x^{2}+y^{2}}<R_{\mathrm{p}}$ by fixing

$$
\begin{aligned}
z & =z(x, y) \\
& =R_{\mathrm{p}}\left[\sqrt{1-\frac{x^{2}+y^{2}}{R_{\mathrm{p}}^{2}}}-\operatorname{atanh} \sqrt{1-\frac{x^{2}+y^{2}}{R_{\mathrm{p}}^{2}}}\right]
\end{aligned}
$$

(v) Find the atomic arrangements with $N \sim \mathcal{O}\left(10^{3}\right)$ that minimize a surface potential energy of the Keating type [32]

$$
\begin{gathered}
E=\frac{3}{16} \frac{\alpha}{a_{\mathrm{CC}}^{2}} \sum_{i, j}\left(r_{i j}^{2}-a_{\mathrm{CC}}^{2}\right)^{2}+ \\
\frac{3}{8} \frac{\beta}{a_{\mathrm{CC}}^{2}} \sum_{i, j, k}\left(\mathbf{r}_{i j} \cdot \mathbf{r}_{i k}+\frac{a_{\mathrm{CC}}^{2}}{2}\right)^{2}+c_{h} \sum_{F_{i}}\left(\left|F_{i}\right|-6\right)^{2}
\end{gathered}
$$

where $\alpha=25.88 \mathrm{eV} \AA^{-2}$ is the bond stretching force constant, $a_{\mathrm{CC}}=1.42 \AA$, $r_{i j}$ is the distance between atoms $i$ and $j$, and $\beta \sim \frac{\alpha}{5}$ is the bond-bending force constant. Finally, the last term favours the formation of hexagonal faces: $F_{i}$ labels the polygons of the net, $\left|F_{i}\right|$ is the number of vertices of the polygons, and ,finally, one has $c_{h}=0.35$ empirically. To reach the energy minimum we repeated the following steps, typically $\mathcal{O}\left(10^{4}\right)$ times:

- Perform random switchings/twists of atomic bonds, based on the Wooten, Winer and Weaire (WWW) method [33] (Fig. 5a); 
a)

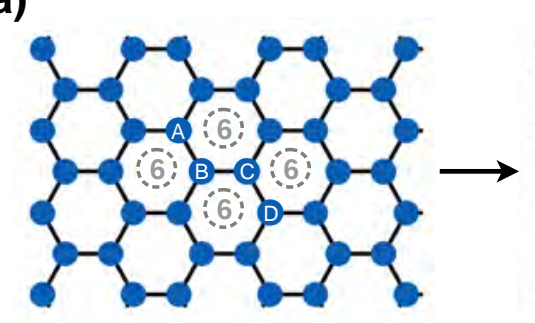

b)

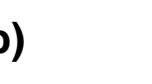
15

- Let the geometry relax through molecular dynamics simulations based on the Fast Inertial Relaxation Engine (FIRE) approach[34];

Figure 5: Bond switch and dualization procedures. a) An example of a bond-switch trial move in a graphene lattice. Starting frơm an initial configuration showing 6-fold rings (dashed circles), the bonds between the carbon atoms $\mathrm{A}-\mathrm{B}$, and $\mathrm{C}-\mathrm{D}$ are cut, and new bonds $\mathrm{A}-\mathrm{C}$ and $\mathrm{B}-\mathrm{D}$ are formed. Twisting then transforms the four 6-fold rings into two 5-fold rings and two 7-fold rings (adapted from Ref. [36]). b) Dualization sequence based on the three-connectivity of the graph. The parent geometry is shown on the left, while the resulting (daughter) geometry is on the right.

- Accept the move only if it lowers the total energy of the system according to the Metropolis algorithm [35].

(vi) Execute on the minimized surfaces a dualization sequence, to increase the number $N$ of atoms and correspondingly the radius of the pseudosphere (Fig. 5b). By using the three-connectivity of the graph one creates a hexagon around each vertex of the initial optimized structure; rescale the bond lengths with a $\sqrt{3}$ factor and repeat from 5 . 


\section{Tight-binding parameter estimate}

Low energy electronic properties of geometries containing millions of atoms, have been evaluated using a TB approach, which is well known to describe correctly the dispersion of graphene around the six Dirac $K$-points in the first Brillouin zone [27]. Due to the pseudosphere curvature, a multi-orbital TB approach has been developed, in which all four valence orbitals $\left(2 s, 2 p_{x}, 2 p_{y}, 2 p_{z}\right)$ are included in the simulation through the Hamiltonian:

$$
H=\sum_{\xi, i} \epsilon_{\xi}^{i} a_{i, \xi}^{\dagger} a_{i, \xi}+\sum_{\xi, \gamma,\langle i j\rangle} t_{\xi, \gamma}^{i j} a_{i, \xi}^{\dagger} a_{j, \gamma},
$$

where $\xi, \gamma$ are orbital label indices while $i, j$ are site indices; $t_{\xi, \chi}^{i j}$ indicates the hopping parameters; $a^{\dagger}$ and $a$ are the creation and annihilation operators; and the symbol $\langle i j\rangle$ means that the nearest neighbours approximation is adopted. The parameters $t_{\xi, \gamma}^{i j}$ describing the hopping between orbitals in different sites were computed within the Slater-Koster formulation [37], which provides a scheme to relate the orbital symmetry, distances and directions of neighbour atoms. Owing to the non-planarity of our geometry we cannot make use of the multi-orbital parametrization typically used for graphene $[38,39]$ where the onsite energy of the $p_{z}$-symmetry orbitals are treated differently from the $x, y$ orbital cartesian components along the in-plane directions (that is $\epsilon_{p_{x}}=\epsilon_{p_{y}} \neq \epsilon_{p_{z}}$ ). Therefore, we derive the TB parameters by fitting abinitio Density Functional Theory (DFT) simulations of the graphene bands by further imposing that the onsite energies for the $p$ orbitals are the same $\left(\epsilon_{p_{x}}=\epsilon_{p_{y}}=\epsilon_{p_{z}}\right)$. DFT simulations of equilibrium and strained configurations of graphene were carried out by using the Quantum Espresso code suite [40]; in particular we use a norm-conserving PBE pseudopotential (C.pbe-mt gipaw.UPF) and an energy cut-off for the wavefuntion expansion on plane-waves set equal to $100 \mathrm{Ry}$. The $k$-point mesh is a $40 \times 40 \times 1$ grid for the calculation of both the ground state density and the band structures. Convergence of the integrals over the Brillouin zone was improved by smearing the occupancy with a $0.136 \mathrm{eV}$ width Gaussian function. The TB parameters that we obtained using Eq. (6) for unstrained $\left(a=a_{\mathrm{CC}}\right)$ and strained $\left(a \neq a_{\mathrm{CC}}\right)$ graphene are: $\epsilon_{s}=-2.8 \mathrm{eV}$ and $\epsilon_{p}=0$ $\mathrm{eV}$ as onsite energies; $V_{s s \sigma}(a)=-5.6 \cdot a / a_{\mathrm{CC}} \cdot e^{-\frac{a-a_{\mathrm{CC}}}{0.55}} \mathrm{eV}, V_{s p \sigma}(a)=5.2 \cdot a / a_{\mathrm{CC}} \cdot e^{-\frac{a-a_{\mathrm{CC}}}{0.75}}$ $\mathrm{eV}, V_{p p \sigma}(a)=4.6 \cdot a / a_{\mathrm{CC}} \cdot e^{-\frac{a_{-}-a_{\mathrm{CC}}}{0.55}} \mathrm{eV}$ and $V_{p p \pi}(a)=-2.44 \cdot a / a_{\mathrm{CC}} \cdot e^{-\frac{a-a_{\mathrm{CC}}}{0.41}} \mathrm{eV}$ as hopping parameters between different orbitals. In Fig. 6 a we report the bands of unstrained and strained graphene obtained by using the DFT and multi-orbital TB approaches.

\section{Kernel Polynomial Method}

For the evaluation of the LDOS we resorted to the KPM, which is a numerical approach useful to access spectral quantities of extended systems for which a direct diagonalization of the full Hamiltonian matrix is computationally unfeasible. It consists in the expansion of the sought quantity in terms of a set of orthogonal polynomials, and then in improving the convergence of the expansion with a kernel to avoid spurious Gibbs oscillations [41]. 
a)
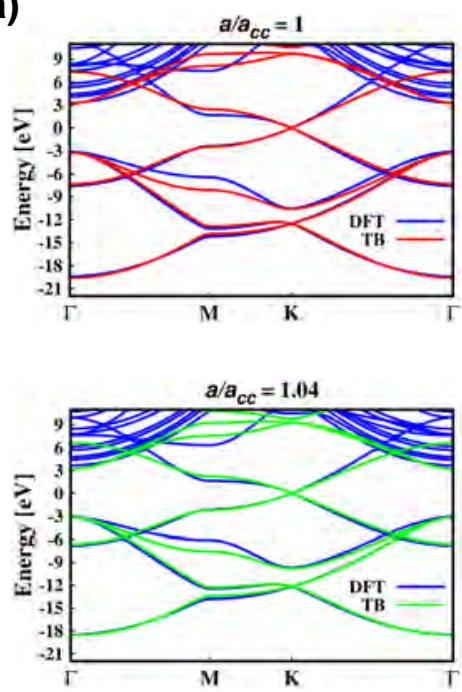

c)
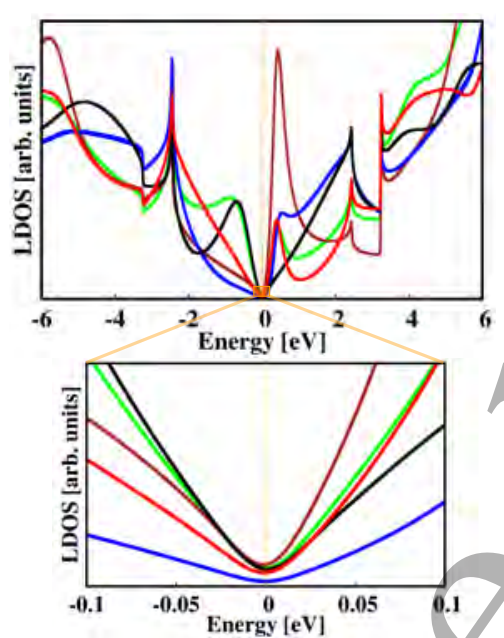

b)

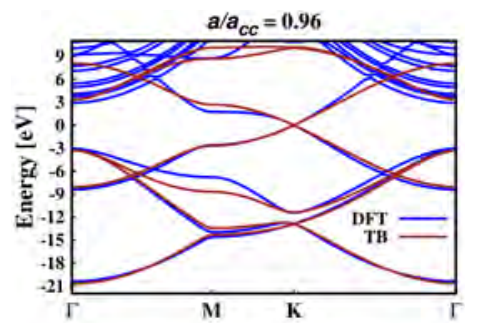

)
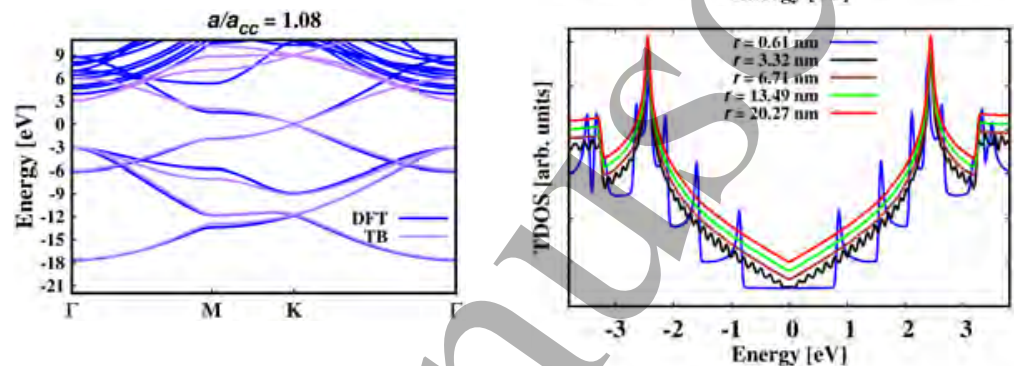

d)
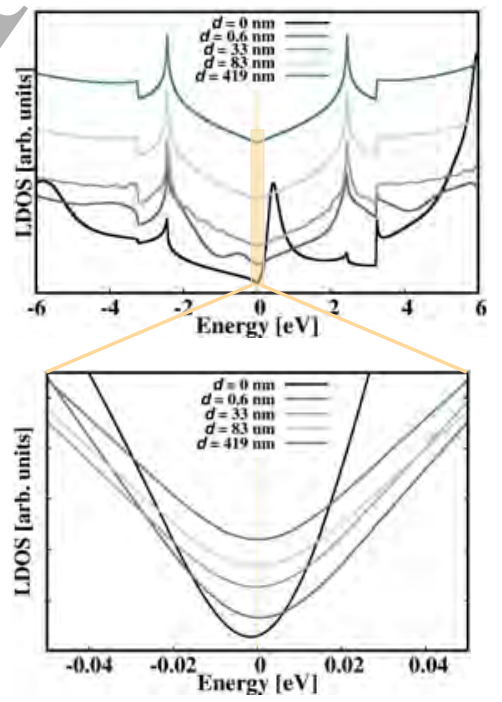

Figure 6: Determination of the parameters for the multi-orbital TB algorithm and corresponding reference LDOS calculations. a) TB fit of graphene bands obtained from DFT electronic structure calculations. From left to right and from top to bottom we report the bands for a biaxial compression of the graphene cell equal to $4 \%$, unstrained graphene, and a biaxial tensile strain of the cell equal to $4 \%$ and $8 \%$, respectively. b) (top) LDOS for different values of the cut-off parameter $N_{c}$ obtained by projection on a site of a pristine graphene rectangular cell with sides equal to $199.97 \mathrm{~nm} \times 199.81$ nm, containing 1,525, 188 carbon atoms; The inset represents a zoom near the Fermi energy. (bottom) total DOS of (n,n) nanotubes for $\mathrm{n}=9(r=0.61 \mathrm{~nm}), n=49(r=3.32$ $\mathrm{nm}), \mathrm{n}=99(r=6.71 \mathrm{~nm}), \mathrm{n}=199(r=13.49 \mathrm{~nm})$ and $\mathrm{n}=299(r=20.27 \mathrm{~nm})$. c) The LDOS angular dependence obtained by projecting over sites belonging to a SW defect (colored atoms in the right panel). d) The LDOS dependence on the radial distance $d$. 
In particular, we used the Chebyshev polynomials for the expansion, and the Jackson kernel to increase convergence, resolution, and accuracy [41]. Within this framework, a generic function can be expanded according to

$$
f(x)=\frac{1}{\pi \sqrt{1-x^{2}}}\left[\mu_{0} g_{0}+\sum_{n=1}^{N_{c}-1} \mu_{n} g_{n} T_{n}(x)\right],
$$

where $T_{n}(x)$ are Chebyshev polynomials of the first kind, $\mu_{n}=\int_{-1}^{1} \mathrm{~d} x f(x) T_{n}(x)$ are the coefficients of the expansion and the $g_{n}$ are the Jackson kernel coefficients defined as

$$
\begin{aligned}
g_{n} & =\frac{1}{N_{c}+1}\left[\left(N_{c}-n+1\right) \cos \frac{\pi n}{N_{c}+1}\right. \\
& \left.+\sin \frac{\pi n}{N_{c}+1} \cot \frac{\pi}{N_{c}+1}\right] .
\end{aligned}
$$

Finally, $N_{c}$ represents the truncation number related to the maximum momentum. The best achievable resolution through this kernel is

$$
\Delta^{J}=\sqrt{1-\cos \frac{\pi}{N_{c}+1}} .
$$

We refer to [41] for the details about the calculation of $\mu_{n}$; here it suffices to emphasize that it is based on the stochastic evaluation of traces, which requires a certain number $R$ of random initial states. As expected, the bigger is $R$, the more accurate becomes the evaluation of the coefficients; we found that $R=100$ was enough for all calculations carried out.

\section{Tests of the LDOS calculations}

The convergence with respect to the $N_{c}$ parameter can be tested in the calculation of the LDOS for the benchmarks cases of planar graphene and armchair carbon nanotubes. The LDOS of graphene for four different values of $N_{c}$ ranging from 2400 to 6000, is shown in the top panel of Fig. 6b). While at a wide energy scale the curves are indistinguishable, zooming near the Fermi energy (set to zero as usual) shows that higher truncation values for $N_{c}$ captures more faithfully the expected linear dispersion relation; on the other hand, there is a threshold to the number of terms in the summation after which spurious oscillations set in, thus spoiling convergence. This can be understood by noticing that the energy separation between levels in periodic graphene is infinitesimal and the DOS is a continuous function. Then, since the pseudosphere in our simulations is a large but finite system and the energy separation of the levels increases with respect to infinite periodic structures, a too big value of $N_{c}$ may result in a KPM energy resolution marginally above the finite energy separation between levels of our finite system, thus leading to poor convergence [42]. For non-planar systems, we have computed the total DOS of $(\mathrm{n}, \mathrm{n})$ nanotubes for $\mathrm{n}=9$, 99, 199 and 299 (radius $r=0.61,3.32,6.71$ and $13.49 \mathrm{~nm}$ ). The total DOS is reported in the bottom panel of Fig. 6b), where we observe that the DOS lineshape of these armchair nanotubes is reproduced surprisingly well already for the moderate value of $N_{c}=2000$ and that, as expected, the confinement 


\section{Exploring Horizons and Hawking Radiation through Graphene Membranes}

effects become less important upon increasing the radius size.

Since a method for estimating the value of $N_{c}$ that trades-off between accuracy and computational efficiency exists only for pristine structures that do not have any defect [42], selecting the best $N_{c}$ is a trial and error process. For the pseudosphere case we found $N_{c}=8000$ to be the optimal value (with $N_{c}=16000$ used when resolving the Fermi energy region in Fig. 4).

\section{LDOS of graphene with a single $S W$ defect}

a)

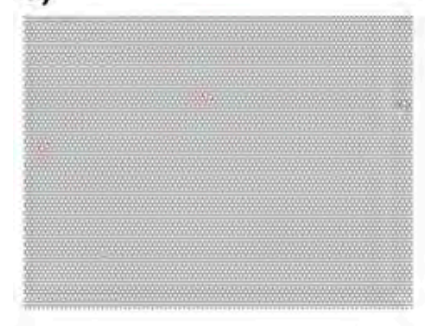

b)

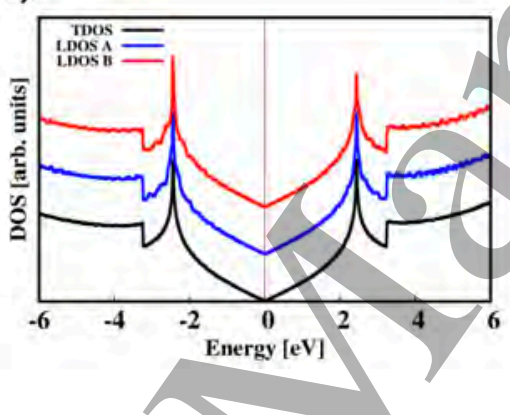

c)

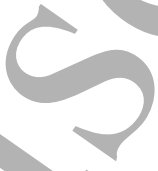

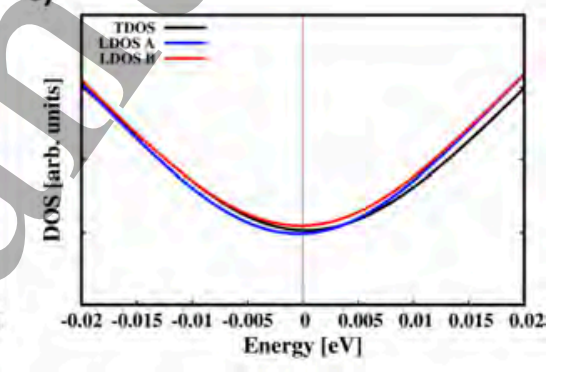

Figure 7: a) Supercell of graphene with $0.05 \%$ density of random SW defects. In red colour we design the atoms belonging to the SW, while the green sphere points out the atomic sites over which we project the LDOS. b) LDOS (red and blue curves) and TDOS (black curve) of graphene with $0.05 \%$ density of random SW defects. c) Zoom near the Fermi energy to show the asymmetric behaviour of the LDOS.

The SW defects are present within the realistic framework of the Beltrami's pseudosphere owing to the negative curvature, while their occurrence is neglected in the analytical continuum model. Thus, we finally investigate the effect of the presence of a single SW defect on the LDOS of a graphene net $(N=823,860)$, particularly near the Fermi energy where our interest is focused. We study both the LDOS projected over different symmetry sites of the SW defect (with $N_{c}=5000$ ), thus obtaining information on the angular dependence (Fig. 6c), as well as the LDOS projected over sites increasingly far from the SW defect, thus obtaining insights on the radial dependence (Fig. 6d). On top of a marked angular dependence, we see that the shape of the LDOS is dramatically modified near the defect site, while far from it the planar graphene shape is recovered; the presence of the SW defect still affects the LDOS projected at distances of $\approx 80 \AA$ with small oscillations in the spectrum. Furthermore, we notice most importantly that near the Fermi energy one observes a marked asymmetry of the LDOS spectrum, persisting again up to a distance of $\approx 80$ $\AA$. This effect overlaps in this energy range and actually is indistinguishable from the asymmetry owing to the negative curvature. 
1

2

3

4

5

6

7

8

LDOS of graphene with a random density of $S W$ defects

The presence of a single SW in a graphene large area is of course unrealistic, while we aim at simulating a surface as close as possible to experimental conditions. Thus, we increased the number of SW defects, which were created randomly within the graphene lattice. In this case, we calculate the DOS projection over sites positioned in the two different sublattices. These simulations were carried out in a graphene lattice containing $N=1,240,236$ carbon atoms, where we introduced 100 SW defects.

A square cut of this planar structure is shown in Fig. 7a). After testing the convergence parameters were set to $R=100$ and $N_{c}=6000$. In Fig. $7 \mathrm{~b}$ ) we show the LDOS (blue and red curves) and the TDOS (black curve) of this structure. The LDOS, in particular, is plotted at a distance of approximately $100 \AA$ from the nearest defect site. Surprisingly, here the LDOS projected over the two sites belonging to the sublattices A and B have a line-shape similar to the single-defect case and to the pristine graphene (see Fig. 6b)) but showing somewhat noisier peaks. On the other hand, the TDOS is smoother, as the contributions of the defects averages out in this case. We observe that an asymmetric behaviour is found again by zooming the LDOS near the Fermi energy (see Fig. 7c)). 